Analysis Method

\title{
Liquid scintillation counting of solid-state plastic pellets to distinguish bio-based polyethylene
}

\author{
Yoshiyasu Nagakawa $^{* 1}$, Shunji Yunoki ${ }^{1}$, Masaaki Saito ${ }^{1}$ \\ ${ }^{1}$ Biotechnology group, Tokyo Metropolitan Industrial Technology Research Institute, \\ Tokyo, Japan \\ * Corresponding author: \\ Biotechnology group, Tokyo Metropolitan Industrial Technology Research Institute \\ 2-4-10, Aomi, Koto-ku, Tokyo 135-0064, JAPAN \\ Tel: $+81-3-5530-2671$ \\ Fax: +81-3-5530-2629 \\ E-mail: nagakawa.yoshiyasu@iri-tokyo.jp
}




\begin{abstract}
Bio-based polyethylene (bio-PE) is chemically identical to conventional fossil-based PE. The only method to differentiate them is radiocarbon $\left({ }^{14} \mathrm{C}\right)$ analysis. The purpose of this study was to evaluate the potential of liquid scintillation counting (LSC) as a screening method. The pellets of bio-PE and fossil-PE were soaked in a scintillation cocktail, and measured by LSC. The counting rate of bio-PE increased as a function of time and then saturated $(35 \pm 1.7 \mathrm{cpm})$ in $30 \mathrm{~h}$. This counting rate was much higher than that fossil-PE $(3.3 \pm 0.5 \mathrm{cpm})$. Pre-soaking of PE in toluene (the solvent of the scintillation cocktail) enhanced the counting rate of bio-PE. Bio-PE with higher crystallinity showed a lower counting rate. These results suggest that the solvent of the scintillation cocktail permeated into the non-crystalline regions, inducing scintillation of bio-PE in the solid state. LSC is a potential high-throughput screening method to detect bio-PE.
\end{abstract}

Keywords: bio-polyethylene, radiocarbon, liquid scintillation counting, screening method, non-crystalline region 


\section{Introduction}

Recently, general-purpose resins, including polyethylene (PE) and polypropylene, (PP) have been synthesized from bio-based monomers instead of petroleum-derived monomers to avoid problems caused by petrochemical plastics [1, 2]. Bio-based PE (bio-PE) has been produced industrially because bio-ethanol can be catalytically dehydrated to ethylene by a proven technology [1]. The production cost of bio-PE is higher than that of fossil-PE, but in some specific cases bio-PE is more beneficial to market because it is regarded as an eco-friendly product made from bio-resources.

Bio-PE is chemically identical to fossil-PE, and an analytical method to distinguish them has been established: the American Society for Testing and Materials (ASTM) D6866-12 [3] describes how accelerator mass spectrometry (AMS) can be used to determine "bio-based content" of products by counting radiocarbon $\left({ }^{14} \mathrm{C}\right)$ content. This method is based on the fact that biological carbon (i.e., carbon in organisms) contains a trace of ${ }^{14} \mathrm{C}$ whereas fossil resources contain no ${ }^{14} \mathrm{C}$. AMS has proved to be a powerful tool to determine bio-based content of plastics [4, 5] and industrial flue gas [6]. Nevertheless, because the AMS apparatus is expensive, uncommon and complicated to operate, a screening method for testing bio-plastics is required.

The purpose of this study is to evaluate the potential of liquid scintillation counting (LSC) as a candidate for such a screening method. LSC is a convenient ${ }^{14} \mathrm{C}$ measurement method that involves soaking samples in a scintillation cocktail and subsequently setting the samples in the apparatus for measurement. Cost of the LSC apparatus is much lower than that of AMS. However, LSC has been considered to be useful only for liquid samples such as biofuels [7-9]. We speculated that bio-PE pellets soaked in a scintillation cocktail would show sufficient liquid scintillation in spite of the insolubility of PE in the cocktail. This assumption is based on the fact that some organic solvents used for scintillation cocktails permeate into amorphous regions of polyolefins [10]. In this study, bio-PE pellets were soaked in a scintillation cocktail and the solid-state samples were measured by a conventional LSC for ${ }^{14} \mathrm{C}$ counting. We demonstrate that LSC is a potential screening method to distinguish bio-PE from fossil-PE. In addition, the mechanism for LSC measurement of solid-state PE is discussed.

\section{Experimental}

2.1. Materials and reagents 
Bio-PE pellets (high-density PE SGF4950, Braskem, Brazil), fossil-PE pellets (high-density PE Novatec HD HJ360, Japan Polyethylene, Japan) and the scintillation cocktail (AL-1; Dojindo, Japan) were the materials used in this study.

To investigate the effect of pre-soaking in an organic solvent, bio-PE pellets $(7 \mathrm{~g})$ were put in a glass vial containing toluene $(20 \mathrm{~mL})$ and then heated in a water bath at $60^{\circ} \mathrm{C}$ for $2 \mathrm{~h}$. To investigate the dissolution of PE in the scintillation cocktail, bio-PE pellets $(20 \mathrm{~g})$ were put in a glass vial containing the scintillation cocktail $(40 \mathrm{~mL})$ and left at $15^{\circ} \mathrm{C}$ for $3 \mathrm{~d}$. The pellets were then removed and ${ }^{14} \mathrm{C}$ content of the residual scintillation cocktail was measured by LSC.

\subsection{Preparation of cylindrical PE specimens with different crystallinities}

The crystallinity of PE was changed by controlling the molding temperature. The bio-PE pellets were put into a metal mold (diameter $15 \mathrm{~mm}$, height $15 \mathrm{~mm}$ ), and placed in a hot press (AH-1TC; As one, Japan) under a pressure of $1 \mathrm{MPa}$. The mold was heated to $200^{\circ} \mathrm{C}$ and held for $30 \mathrm{~min}$ to melt the pellets. The mold was subsequently cooled by a slow-cooling process whereby the power of the hot press was turned off while maintaining pressure and left overnight, or by a fast-cooling process whereby the mold was soaked in an ice bath for $5 \mathrm{~min}$. After conducting the respective cooling process, cylindrical specimens of varying crystallinity were obtained.

The crystallinities of the cylindrical bio-PE samples were determined by differential scanning calorimetry (DSC) (DSC-60; Shimadzu, Japan) according to a previously reported method [11] with slight modifications. In brief, 5-6 mg samples were heated from $50^{\circ} \mathrm{C}$ to $160^{\circ} \mathrm{C}$ at $10^{\circ} \mathrm{C} / \mathrm{min}$, and then cooled at $10^{\circ} \mathrm{C} / \mathrm{min}$. The crystallinity $\left(\chi_{c}\right)$ of the specimen was calculated by equation (1).

$$
\chi_{c}=\left(\Delta H_{\text {exp }} / \Delta H\right) \times 100 \%
$$

where $\Delta H_{\text {exp }}$ is the experimental heat of fusion and $\Delta H$ is the assumed heat of fusion of fully crystalline HDPE (293 J/g).

\subsection{LSC measurements}

LSC measurements of PE were carried out using a Tri-Carb 3180 TR/SL (PerkinElmer; Waltham, USA) in the ${ }^{14} \mathrm{C}$ normal counting mode. Channel windows 
(5.0-156.0 keV) were applied to exclude a contribution from dark current or chemiluminescence. PE pellets ( $7 \mathrm{~g})$ or the cylindrical PE $(6 \mathrm{~g})$ and scintillation cocktail $(10 \mathrm{~mL})$ were put in an LSC glass vial, and then placed in the LSC apparatus. Counting for 50 min was repeated 99 times (for a total of $83 \mathrm{~h}$ ), and the counting rate (counts per minute: $\mathrm{cpm}$ ) was recorded.

\section{Results}

To evaluate the dissolution of bio-PE in the scintillation cocktail, we measured the residual scintillation cocktail obtained from the pre-soaking experiment of bio-PE pellets at $15^{\circ} \mathrm{C}$ for $3 \mathrm{~d}$. The temperature was set to be equal to that of the sample chamber of the LSC apparatus. There was no significant difference in the counting rates between the samples and fresh scintillation cocktail, indicating that bio-PE was not dissolved.

The ${ }^{14} \mathrm{C}$ content of the fossil-PE and bio-PE pellets were measured by LSC. The counting rates of the bio-PE increased depending on the amount of time the pellets were soaked in the scintillation cocktail, which nearly saturated after $30 \mathrm{~h}$ (Fig. 1). The saturated counting rate of bio-PE ( $35 \pm 1.7 \mathrm{cpm}$ [mean $\pm 2 \sigma$; for $50 \mathrm{~min}])$ was much higher than that of fossil-PE $(3.3 \pm 0.5 \mathrm{cpm})$. A LSC measurement was also carried out for the bio-PE pellets, which were pre-soaked in hot toluene at $60^{\circ} \mathrm{C}$. The initial counting rate $(27 \pm 1.5 \mathrm{cpm})$ was significantly higher than that of bio-PE without pre-soaking (7.9 $\pm 0.8 \mathrm{cpm}$ ) (Fig. 1). The saturated counting rate of the pre-soaked bio-PE was also higher than that without pre-soaking (Fig. 1).

The bio-PE was molded into a cylindrical shape at different cooling rates, and the crystallinities $\left(\chi_{c}\right)$ were determined by DSC. The Bio-PE $\chi_{c}$ prepared by slow-cooling and fast-cooling were determined to be $73 \%$ and $52 \%$, respectively. The counting rates of the LSC measurements of bio-PE prepared by slow-cooling and fast-cooling were 1.0 $\pm 0.01 \mathrm{cpm} / \mathrm{g}$ and $1.2 \pm 0.01 \mathrm{cpm} / \mathrm{g}$, respectively.

\section{Discussion}

The focus of our research was to evaluate LSC measurements of PE pellets to screen bio-PE prior evaluation by AMS. PE was not dissolved in the scintillation cocktail consisting of toluene, detergents and scintillators. This fact defines the LSC measurement of PE pellets as a "solid-state LSC measurement." LSC is a specialized method for sample measurement that can be solubilized or emulsified in a scintillation 
cocktail to achieve a uniform sample distribution. As such, solid-state LSC measurements have been neglected or considered to be unsuccessful. Contrary to this consideration, we demonstrated that bio-PE pellets soaked in a scintillation cocktail showed significantly higher counting rates than that of fossil-PE. This result suggests that bio-PE can be simply screened by soaking pellets in a scintillation cocktail with subsequent LSC measurements.

A possible explanation for the successful results determined for solid-state LSC is that the solvent (toluene and a small amount of xylene) permeated into noncrystalline regions of the PE, which caused scintillation analogous to liquid scintillation. This mechanism is supported by the following findings: presoaking of bio-PE in toluene accelerated the time-dependent increase in the counting rate, and the counting rate of cylindrical bio-PE with lower crystallinity was higher than that with higher crystallinity. If such solvent permeation did not occur, sufficient counting rates could not have been obtained because scintillation occurs only around the surface of pellets. The average range of ${ }^{14} \mathrm{C}$-derived $\beta$-rays $\left(\mathrm{E}_{\max }=156 \mathrm{keV}\right)$ in $\mathrm{PE}$ is about $40 \mu \mathrm{m}$ [12], indicating that most of the ${ }^{14} \mathrm{C}$ in the pellets (approximate diameter of $3 \mathrm{~mm}$ ) does not contribute to LSC measurements. On the assumption that $\beta$-rays emitted from ${ }^{14} \mathrm{C}$ which exists at a depth of $0-40 \mu \mathrm{m}$ contribute to LSC, the counting rate of bio-PE pellets $(7 \mathrm{~g})$ would be estimated to be $3.25 \mathrm{cpm}$. This value is insufficient to distinguish bio-PE from fossil-PE. The results suggest that solvent permeation could play a key role in solid-state LSC measurements.

Pre-soaking in toluene proved to be useful to accelerate the solvent permeation and the resulting increase in counting rates. Counting rates and LSC times are important factors for successful screening. As counting rates increment, counting errors diminish in significance, and the accuracy of the LSC method is thereby increased. High-throughput screening depends greatly on counting times because the LSC apparatus cannot measure multiple samples simultaneously. Assuming isolated screening required $3 \mathrm{~h}$, pre-soaking for $1 \mathrm{~h}$ would decrease measurement times to only 2 h.

We must note that it may be difficult to determine bio-PE/fossil-PE ratios precisely in their composites by LSC measurements. Samples may have various sizes, shapes and crystallinities in practical measurements, and our results indicate that those factors affect the results of solid-state LSC measurements. In addition, manufactured PE is frequently colored with various dyes. It is known that the accuracy of LSC measurements drops because of "color quenching." To establish solid-state LSC measurements as a reliable screening method, sample processing to cancel sample 
history are important issues.

In conclusion, solid-state LSC measurements of PE pellets can distinguish bio-based from fossil-based materials, and this method represents a possible screening method for AMS. The measurement process for solid-state LSC is simple: soak the PE pellet in a scintillation cocktail and then set the sample in the LSC apparatus for measurement. In the near future, we will report on sample processing methods to minimize the influences of factors affecting solid-state LSC measurements (such as pellet sizes, shapes, and crystallinities).

\section{Acknowledgments}

Bio-based polyethylene was kindly provided from Toyota Tsusho Corporation. The authors thank Mr. Toshiyuki Yamanaka for his assistance on DSC measurements. This work was partially supported by a grant program "Adaptable and Seamless Technology Transfer Program through target-driven R\&D (A-STEP), Exploratory Research, JST." The authors would like to thank Enago (www.enago.jp) for the English language review. 


\section{References}

[1] G. Chen, M. K. Patel, Plastics Derived from biological sources: present and future: a technical and environmental review, Chem. Rev. 112 (2012) 2082-2099.

[2] A. U. B. Queiroz, F. P. Collares-Queiroz, Innovation and industrial trends in bioplastics, Polym. Rev. 49 (2) (2009) 65-78.

[3] ASTM International, ASTM D6866-12 Standard test methods for determining the biobased content of solid, liquid, and gaseous samples using radiocarbon analysis, 100 Bar Harber Drive, West Conshohocken, Pa. USA (2012)

[4] Y. Tachibana, T. Masuda, M. Funabashi, M. Kunioka, Chemical synthesis of fully biomass-based poly(butylenes succinate) from inedible-biomass-based furfural and evaluation of its biomass carbon ratio, Biomacromolecules, 11 (2010) 2760-2765.

[5] T. Onishi, F. Ninomiya, M. Kunioka, M. Funabashi, K. Ohara, Biomass carbon ratio of polymer composites included biomass or petroleum origin resources, Polym. Degrad. Stab. 95 (2010) 1276-1283.

[6] S. Yunoki, M. Saito, Y. Nagakawa, Influence of atmospheric ${ }^{14} \mathrm{CO}_{2}$ on determination of the ratio of biogenic carbon to fossil one in exhaust gases using accelerator mass spectrometry: experimental evaluation for industrial flue gases, RADIOISOTOPES, Vol.61, No.6 (2012) 307-313.

[7] S. Yunoki, M. Satio, A simple method to determine bioethanol content in gasoline using two-step extraction and liquid scintillation counting, Bioresour. Technol. 100 (2009) 6125-6128.

[8] M. Saito, M. Nakamura, Easy C-14 measurement of bio-gasoline E3 by extraction with water, RADIOISOTOPES, Vol.56, No.7 (2007) 383-385.

[9] M. Saito, M. Nakamura, Easy C-14 measurement of bio-gasoline E3 by double-extraction with water, RADIOISOTOPES, Vol.56, No.9 (2007) 529-531.

[10] T.V. Prasad, K.W. Brown, J.C. Thomas, Diffusion coefficients of organics in high density polyethylene (HDPE), Waste Manag. Res. 12 (1994) 61-71.

[11] Y. Lei, Q. Wu, C. M. Clemons, F. Yao, Y. Xu, Influence of nanoclay on properties of HDPE/Wood composites, J. Appl. Polym. Sci. Vol. 106, (2007) 3958-3966.

[12] ICRU, ICRU report 37: Stopping powers for electrons and positrons, ICRU, Bethesda, USA (1984), pp. 178 


\section{Figure captions}

Fig 1: Time-dependent change of counting rates obtained by LSC measurements of PE pellets. Open circles represent bio-PE pellets pre-soaked in hot toluene at $60^{\circ} \mathrm{C}$. Closed circles represent bio-PE pellets without pre-soaking. Closed triangles represent fossil-PE pellets as a background. 
